\title{
Exploiting the FM-signal in a laser-diode SMI by means of a Mach-Zehnder filter
}

\author{
Michele Norgia, Senior Member, IEEE, Dario Melchionni, and Silvano Donati, Life Fellow, IEEE
}

\begin{abstract}
We develop a new method to read the frequency modulation (FM) of a laser diode (LD) self-mixing interferometer (SMI), based on using a Mach-Zehnder interferometer as an edge filter, and obtain improved performance respect to the normally used amplitude modulation (AM) signal. The converted FM signal is much larger and has a better SNR than the normally used AM signal. The minimum detectable signal of the SMI is improved by a factor 150 with our setup that uses an all-fiber technology to realize the compact Mach-Zehnder filter (MZF). The MZF has a record $19(\mathrm{GHz})^{-1}$ conversion factor and is tuned at half-fringe on the laser wavelength with a feedback loop acting on the laser bias current. On small amplitude vibrations measured with the converted-FM signal, we attain a displacement (NED) of $1.3-\mathrm{pm} / \sqrt{\mathrm{Hz}}$, about two order of magnitude better than the AM channel or a normal SMI.
\end{abstract}

Index Terms - Interferometry, Optical Instruments, Laser Sensors

\section{INTRODUCTION}

S elf-mixing interferometry (SMI) in laser diodes (LD) is a wellknown measurement technique [1-3], used in instrument for vibration and displacement analysis of a remote target [4-11], and for the measurement of LD parameters [12-13]. SMI has been employed successfully in industrial [14-17], biomedical [18,19] and consumer applications [20].

In an SMI, the weak optical back-reflection from a remote target reentering the LD cavity perturbs the optical field, generating modulations of it. The resulting interaction is nicely described by the well-known Lamb's equations [21], or their extension to the diode laser, that is, the Lang and Kobayashi equations (LKE) [22]. From theory, we find that the cavity field undergoes modulations of both amplitude (AM) and frequency (FM). Because impressed on power, the AM signal is readily detected by the LD monitor photodiode, and it can be found everywhere down the laser beam, although the signal polarity may change [23, 24] from front to rear mirror outputs. Another possibility is to tap the AM signal as the voltage across the LD terminals, an option particularly useful for $\mathrm{THz}$ wavelengths [25] because it dispenses with the photodiode at the expense of a loss in the signal-to-noise ratio (SNR) [26].

On the other hand, the FM signal was practically left unused in all reported applications, because not directly accessible in a laser diode.

Recently, Contreras et al. [27] proposed a technique for detecting the FM signal, using a low-pressure acetylene-cell filter as a steep-edge

Manuscript received XXXXX, 2017, revised xxx, 2017, accepted xxx, 2017. M. Norgia and D. Melchionni are with the Department of Electronics, Informatics and Bioengineering of Politecnico di Milano, p.za L. da Vinci 32, Milano. S. Donati is with the Department of Industrial and Information Engineering, University of Pavia, v. Ferrata 1, 27100 Pavia, Italy.

Corresponding Author: Michele Norgia e-mail: michele.norgia@polimi.it $(\approx 4$-pm) frequency discriminator centered at $\lambda=1531.58 \mathrm{~nm}$ and providing a FM-to-AM conversion factor of $2.17(\mathrm{GHz})^{-1}$. With the setup, both amplitude and signal-to-noise ratio were found much larger than those of a conventional AM [27], hinting at the use of converted FM signal to develop an SMI with improved performance.

However, some drawbacks are apparent in using a gas cell as filter: first, it is a fixed-wavelength device and requires a laser emitting at a wavelength matched to the filter, a bound to instrumental flexibility; second, the conversion factor is limited to a few $(\mathrm{GHz})^{-1}$ by the cell absorption line-width, even at low gas pressures; third, the cell is relatively bulk size (typ. 165-mm long) compared to the very compact diode-laser of the plain SMI setup. Last, a questionable detail of the experiment in Ref. [27] is the use of a high power laser source, a DFB integrated in a master-oscillator power-amplifier with a $350-\mathrm{mW}$ emitted power.

To overcome the above problems, we propose using a Mach-Zehnderinterferometer filter (MZF) as the selective element providing a high FM-to-AM conversion factor. The MZF is compact and, thanks to the periodic response in frequency, the wavelength working range is virtually unlimited, the selectivity is easily tailored to the desired value by selecting the appropriate path-length difference of the two arms, and last but not least, tuning is easily achieved by path-length control of one arm.

In this letter, we develop the all-fiber implementations of the MZF, and present the measurement results of amplitude and noise of both AM and converted-FM signals. Performances of a vibrometer using the converted-FM signal are finally reported.

\section{ANALYSIS}

As a starting point, let us consider the SMI operation on a remote target placed at a distance $d$, and write the frequency $v$ of the perturbed oscillating field by solving the Lang-Kobayashi Equations (LKE) [22] in terms of the external round trip time-of-flight $\tau_{\text {ext }}=2 \mathrm{~d} / \mathrm{c}$, where $c$ is the speed of light. After some algebra, we get from the LKE the wellknown result $[1,2]$ :

$$
2 \pi\left(v-v_{0}\right) \tau_{\text {ext }}=-C \sin \left(2 \pi v \tau_{\text {ext }}+\operatorname{atan} \alpha\right)
$$

where $v_{0}$ is the unperturbed laser frequency, $C$ is the feedback parameter and $\alpha$ is the line-width enhancement factor.

From Eq. 1 we can see that the FM signal $\Delta v=v-v_{0}$ of the SMI is a sine function of the phase $2 \pi v \tau_{\text {ext }}=2 k d$, and has a (peak) amplitude $\Delta v_{p}$ given by:

$$
\Delta v_{p}=C / 2 \pi \tau_{e x t}
$$

As the perturbed frequency $v$ is not much different from the unperturbed $v_{0}$, the FM signal is a $2 \pi$-periodic function of the argument $2 \pi v_{0} \tau_{\text {ext }}$. Also, on letting $\tau_{\text {ext }}=2 d / c$ and $2 \pi v_{0} / c=k_{0}$, it is 
seen a $2 \pi$-periodic function of the external optical phase shift $2 k_{0} d$. The FM signal makes a full cycle of variation for $2 k_{0} \Delta d$ $=2 \pi$, that is, for a distance change $\Delta d=\lambda / 2$, as in a normal interferometer and also in an SMI based on the AM signal [1].

To get an order of magnitude for $\Delta v_{p}$, we can assume a moderate feedback regime [1] for the SMI, that is $C \sim 1$, and take $d \sim 1 \mathrm{~m}$ as the typical target distance. Then we get $\tau_{\text {ext }}=2 d / c=6.7 \mathrm{~ns}$, and the corresponding peak FM signal is $\Delta v_{p} \sim 25 \mathrm{MHz}$. This value is used below as reference for the design of the MZF.

\section{EXPERIMENTS}

In Fig. 1 we report the setup realized in all-fiber technology. At the output of the laser diode (LD) we split the beam into two branches: the main one ( $95 \%$ of the power) is directed to the remote target under measurement, and the second one (5\% of the power) is used for the readout of the FM signal.

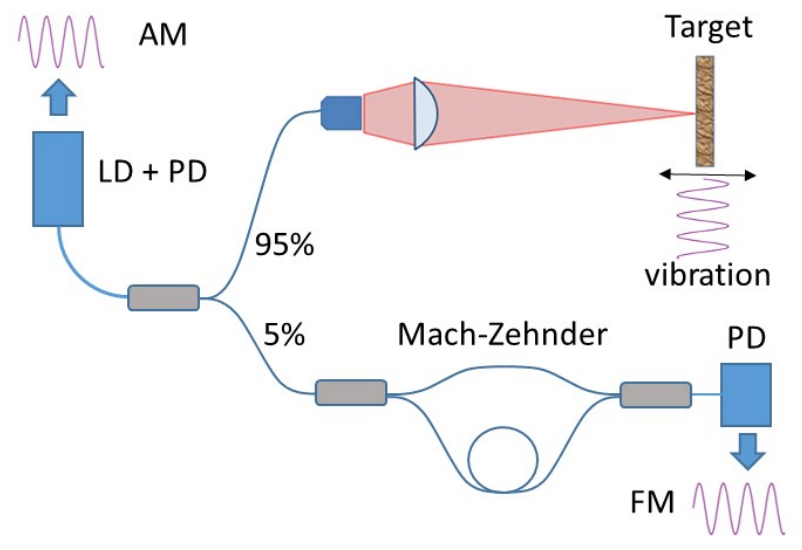

Fig.1. All-fiber setup for detecting the frequency modulation (FM) signal of the Self-Mixing Interferometer: a small fraction (5\%) of the power from the laser diode (LD) is sent to the Mach-Zehnder filter for the frequency-toamplitude conversion. The main output branch ( $95 \%$ power) goes to a fiber collimator and objective lens, projecting the spot on the target under measurement. The monitor photodiode of the laser (group LD+PD) supplies the usual amplitude modulation (AM) signal for comparison.

As a source, we employed a commercial laser diode for optical communication, a $\lambda=1550 \mathrm{~nm}$ DFB of Wavespectrum (WSLD- 1550$020 \mathrm{~m}$ ) with $20-m W$ output power in air and $8-m W$ in the fiber pigtail. The laser feeds two branches. In the measurement branch (the 95\% coupler output) the laser beam is sent to the target (a loudspeaker with white paper surface) through a fiber collimator and a focusing lens. For the FM-conversion branch, a small fraction $(5 \%)$ of the $8-m W$ output of the pigtailed laser is directed to the MZF. Unwanted reflections are reduced by means of Angled Physical Contact (APC) connectors. The frequency-to-amplitude conversion is performed by the MZF with a selected optical length imbalance $\Delta L$ between the two arms. The incoming beam, split by means of a $3-d B$ coupler, travels down the two paths and then recombines through a second $3-d B$ coupler.

Due to the path-length imbalance, the transmission $T$ of the MZF is a periodic function of frequency $v$ and is written as:

$$
T(\lambda, \Delta L)=1 / 2[1+\cos (2 \pi v n \Delta L / c)]
$$

where $n$ is the effective index of refraction of the fiber.
The frequency periodicity $\Delta v_{M Z}$ of the MZF is found by letting $2 \pi \Delta v_{M Z} n \Delta L=2 \pi$ in Eq.3, with the result $\Delta v_{M Z}=c / n \Delta L$.

About the conversion of the FM signal $\Delta v_{\mathrm{p}}$ into an amplitude signal $\Delta P_{F M}$, for small amplitudes we have simply a linear regression, $\Delta P_{F M}$ $=P_{0} S \Delta v_{p}$, where $S=d T / d v$ is the slope of the transmission response, and $P_{0}$ is the optical power. On biasing the MZF in the middle of the transmission curve (that is, at $T=1 / 2$, or at half-fringe), the slope of the transmission curve $S$ is maximum and its value is found from Eq. 3 as:

$$
S=d T / d v=2 \pi n \Delta L / c
$$

For an imbalance $n \Delta L=90-\mathrm{cm}$ (corresponding to $\Delta L \approx 60-\mathrm{cm}$ of fiber), Eq.4 gives $S=19(\mathrm{GHz})^{-1}$, a value good enough to supply a large amplitude of the converted signal $S \Delta v_{p}$ while keeping the signal modulation $\Delta v_{p} \sim 25 \mathrm{MHz}$ small with respect to the total swing of the MZF response (see Fig.2). As the periodicity in frequency of the MZF is $\Delta v_{M Z}=c / n \Delta L \sim 330 \mathrm{MHz}$, the maximum (integral) linearity error for a $25-\mathrm{MHz}$ swing turns out to be a modest $7.6 \%$, so we can dispense with an a-posteriori correction.

Interesting to note, we obtain a slope $S$ about one order of magnitude larger the $2.17(\mathrm{GHz})^{-1}$ of the $16.5-\mathrm{cm}$ long acetylene cell of Ref. [27], with a smaller size of the packaged all-fiber filter (60- $\mathrm{mm}$ long), and without constraint on wavelength. For increased distance and smaller $\Delta v_{p}$ we can make an even larger imbalance $n \Delta L$ in the MZF, and conversion factor $S$ up to $100(\mathrm{GHz})^{-1}$ are feasible with limited $(<5 \%)$ linearity error.

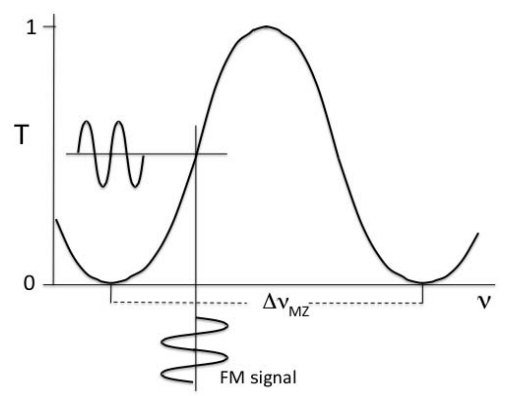

Fig.2. Schematic representation of the MZI filter response and of the conversion of the FM signal.

The output of the Mach-Zehnder is fed to the photodiode (PD in Fig.1), a commercial module with $125-\mathrm{MHz}$ bandwidth and overall transresistance $40-k \Omega$. The AM signal is measured on the LD monitor photodiode by an amplifier with the same $R_{F}=40-k \Omega$ response, but a bandwidth limited to $28 \mathrm{kHz}$, to filter out the larger noise of the AM channel.

Fig. 3 shows an example of measurement: the converted FM signal amplitude is read as $1.4 V_{p p}$ and the noise floor is $15 \mathrm{mV} r m s$, on a bandwidth $20 \mathrm{MHz}$ (as limited by the oscilloscope). The AM signal amplitude is $\approx 20 \mathrm{mV}$ p , and the noise floor is $\approx 1.2 \mathrm{mV} V_{r m s}$, on a $28-\mathrm{kHz}$ bandwidth.

Thus, at the amplifiers' outputs, the converted FM signal is 1.4/ $/ 20 m=70$ times larger than the AM. The ratio of collection efficiency of the photodiodes is measured $\eta_{F M} / \eta_{A M}=1.18$ while the transfer factors of the remaining electronic chain are equal. Accounting also for the $95 / 5=19$ optical splitter ratio, the effective FM-to-AM gain sums up to $G_{A}=70 \cdot 19 / 1.18=1127$, at equal powers in the channels.

Even more important, the SNR of the FM signal, $1.4 / 15 m=93.3$, is larger than the AM signal, 20/1.2m=16.7 and, accounting also for the 
bandwidth square-root ratio dependence, $(20 M / 28 k)^{1 / 2}=26.72$, the improvement in SNR for the converted-FM respect to the AM channel totals to $G_{S N R}=149$ (or $43.4 \mathrm{~dB}$ ). [A detailed analysis of amplitude and SNR gains is outside the scope of this letter and will be developed in a forthcoming paper].

These results show the same magnitude of those reported previously, but are obtained with much smaller optical power ( $8-\mathrm{mW}$ compared to $230-m W$ of Ref. [23]) suitable for mitigated laser safety requirements.

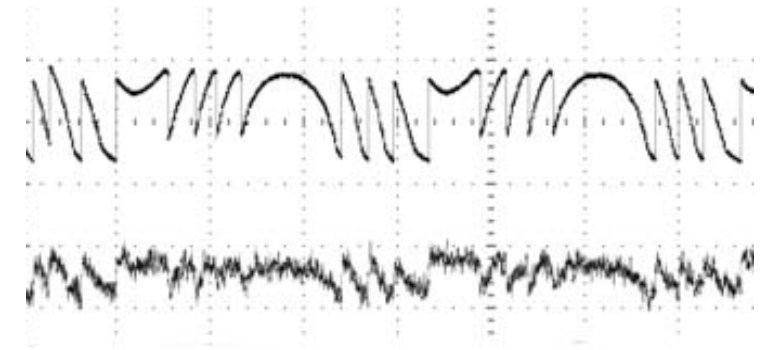

Fig.3. SMI signals from the all-fiber setup of Fig.1. Top trace is the converted-FM signal, scale 5V/div, bottom the AM signal, scale $20 \mathrm{mV} / \mathrm{div}$. Target drive is a sine wave of 3.3-ms period.

About signals, two remarks are in order. First, the AM signal is picked at the rear mirror of the laser (group LD+PD in Fig.1), but is present also at the front output, so it is invariably summed up to the (much larger) converted FM signal at the filter output. Yet, in view of the large ratio of the two signals, the AM contribution can be neglected. Second, the AM and FM signal look almost in-phase in Fig.3 (while usually they are a sine/cosine pair [1]) but this is not a surprise because the phase-shift is $\Phi_{A M-F M}=\pi / 2$-atan $\alpha$ [30], and for our diode laser the $\alpha$ factor is found about 6 (using the waveform inspection method [13]).

In the all-fiber experimental setup, the main trouble was the drift of the MZF working point with temperature and ambient microphonics. To remove the drift, we arranged a feedback loop to dynamically lock the interferometer to half-fringe. The loop includes an amplifier, reading the error signal as difference between the external PD output (FM signal) and a reference voltage (1.5- $V$ in our setup), and a drive feed-through to the LD bias current. With a large feedback loop gain $\left(G_{\text {loop }} \approx 200\right.$ in our case) the MZF output is locked to a mean value of $1.5-V$ and any source of drifts, including the LD wavelength, is strongly reduced (by a factor $G_{l o o p}$ ). \{A more effective control scheme would be to use both outputs of the MZF coupler and arrange a balanced detection, thus doubling the signal amplitude and removing commonmode drifts [28], but in our setup this variant was found not necessary\}. Loop bandwidth is limited to $10-H z$ to ensure loop stability, and yet be effective to cancel out slow fluctuations of $n \Delta L$. No selection of the feedback polarity is necessary, as the MZF automatically jumps on the fringe slope corresponding to negative feedback.

The fiber drift-related problems are much mitigated when the fiber is packaged. We experimented it by mounting all the fiber devices in a pre-package box of $60 \times 30 \times 10 \mathrm{~mm}^{3}$. Respect to a loose fiber, with the packaged layout the control of the LD current became lighter, and the all-fiber version proved to work fine and be satisfactory in term of performance and complexity.

\section{SMI VIBROMETER BASED ON THE CONVERTED-FM}

To demonstrate the improvement gained with the cFM approach, we have evaluated the performance in an instrumental application: a laser vibrometer for the measurement of sub-nm amplitudes of oscillation of a remote target.

In Fig.4 we report the exemplary waveforms of cFM and AM signals in a vibration measurement performed on a piezo target, with an untreated (diffusive) surface, placed at $L=80$-cm distance.

First, the piezo response (in $\mu \mathrm{m} / V$ ) was calibrated at large amplitudes (several wavelengths) by counting the $\lambda / 2$ periods developed in a cycle of vibration, then the piezo was driven at nanometer amplitudes with a dc offset adjusted for maximum sensitivity (that is, at half-fringe).

The results in Fig.4 show the dramatic improvement in sensitivity of the vibrometer measurement on measuring a peak-to-peak oscillation of $0.7-\mathrm{nm}$ at $10-\mathrm{kHz}$. The acquisition is digitally filtered with $1-\mathrm{kHz}$ bandwidth. We use the $N E D=\phi_{n} / 2 k$ (noise equivalent displacement) $[1,31]$, or its spectral density $N E D_{S D}=\phi_{n} / 2 k B$ to describe the vibrometer sensitivity.

The AM channel $N E D_{S D}$ is about 220-pm/ $/ \mathrm{Hz}$, and on a bandwidth of 1-kHz this noise corresponds to a minimum detectable amplitude of about $6 \mathrm{~nm}$. As shown in Fig.4, even after a 1-kHz filtering, in the AM channel the 0.7- $\mathrm{nm}$ vibration amplitude is completely hidden in noise. On the contrary, the cFM signal with a $N E D_{S D}$ of about $1.3-p m / \sqrt{ } H z$, measures the vibration well, showing a noise level of about $40-p m$ rms on a $1-k H z$ bandwidth. Thus, with a marginal $S N R$, of say 3 , the minimum detectable vibration amplitude attained by the cFM-based vibrometer is a record 120-pm.

As a reference, state-of-the-art of vibrometers based on a plain SMI are typically positioned at a $N E D_{S D}$ of $50 \ldots 100 \mathrm{pm} / \sqrt{ } \mathrm{Hz}$ at $\lambda=800 \mathrm{~nm}$ and for laser sources of $1 . . .2-\mathrm{mW}$ [29]. At $\lambda=1550 \mathrm{~nm}$ the expected $N E D_{S D}$ is about twice as much, because of the wavelength ratio, and therefore the value of $220 \mathrm{pm} / \sqrt{ } \mathrm{Hz}$ measured with the AM channel of our all-fiber setup is in line with the state-of-the-art results. Thus, the minimum detectable amplitude for a vibrometer based on the AM channel is about 6.6-nm in the same conditions noted above.
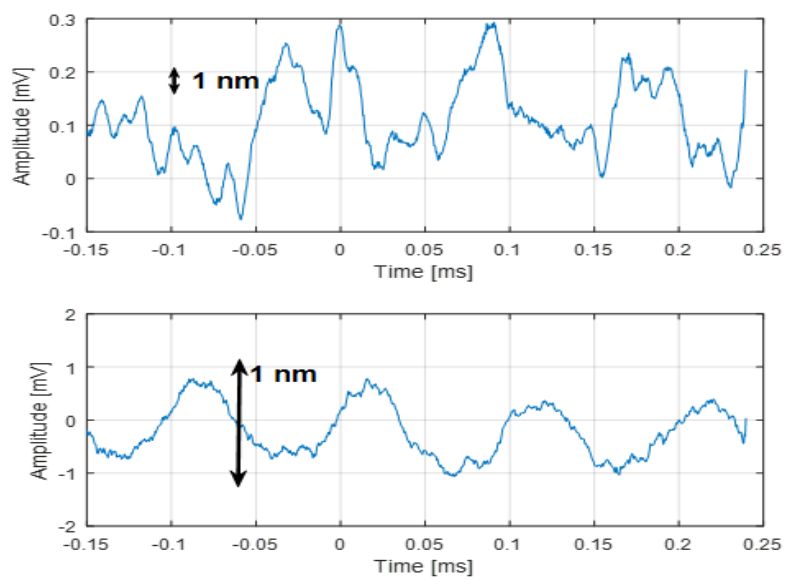

Fig.4. Exemplary waveforms of the AM (top) and converted-FM (bottom) signals, obtained with the setup of Fig.1, in the measurement of a $10-\mathrm{kHz}$ vibration of a diffusive target with $0.7-\mathrm{nm}$ peak-to-peak amplitude generated by a piezo placed at $\mathrm{L}=80-\mathrm{cm}$.

Yet, the quantum limited performance $N E D_{S D q}=(S N R 2 \mathrm{kB})^{-1}$ $[1,31]$, is much smaller, it is calculated as $N E D_{S D q}=0.1 \mathrm{pm} / \sqrt{ } \mathrm{Hz}$ for $a$ $0.35-\mu W$ received power from the diffusive surface at $L=80-\mathrm{cm}$ (and at $\lambda=1550 \mathrm{~nm}$ ), so it would be a 9-pm minimum detectable vibration amplitude (at $S N R=3$ and $B=1-k H z$ ).

The $\mathrm{cFM}$ channel vibrometer is positioned at $N E D_{S D}=1.3 \mathrm{pm} / \sqrt{ } \mathrm{Hz}$, so 
it approaches much better the quantum limit than the SMI AM channel and also than a traditional interferometer for the same audio frequency vibration.

Another remarkable feature is the much higher frequency of operation that can be covered by the cFM channel, up to tens of $M H z$ 's compared to the $k H z$ 's of the AM channel.

The improvement in SNR can be obviously exploited in a number of applications of SMI other than the vibrometer, but developing the details of each application requires further work and is beyond the scope of this Letter.

Finally, we have also tested a free-space counterpart of the scheme in Fig.1, with fiber components replaced by micro-optics components. The resulting setup requires accurate alignment of all the optical components, but has the advantage of a higher power available (20$m W$ ), as the $\approx 8-d B$ loss of fiber pigtailing is cleared, and obviously hasn't the drifts in polarization state of the fiber. Using $n \Delta L=90-\mathrm{cm}$, we were able to obtain a more than doubled SNR improvement of the converted-FM with respect to the AM signal, $G_{S N R}=372$ (or 51.4- $d B$ ) at the expense of a bulkier setup, however.

\section{CONCLUSIONS}

A novel scheme based on a MZF for readout of the FM in SMI is proposed and demonstrated. The filter has increased conversion factor with respect to a gas cell, can be tailored for operation at any wavelength of the SMI laser source, and offers a more compact setup. Even using a modest optical power, the converted-FM SMI outperforms the conventional SMI based on the AM signal in amplitude and in SNR. Improvement of two order of magnitude in minimum detectable signal has been demonstrated in the operation of the SMI as a vibrometer.

\section{ACKNOWLEDGEMENT}

Authors wish to thank Dr. Davide Bellazzi and Dr. Alessio Balbo for their invaluable technical support in the experiments.

\section{REFERENCES}

[1] S. Donati, "Developing Self-Mixing Interferometry for Instrumentation and Measurements," Laser Photonics Rev. 6, 393 (2012).

[2] G. Giuliani, M. Norgia, S. Donati, and T. Bosch, "Laser diode self-mixing technique for sensing applications," J. Optics A 4, S283 (2002).

[3] T. Taimre, M. Nikolic, K. Bertling, Y.L. Lim, T. Bosch, and A. Rakic, "Laser feedback interferometry: a tutorial on the self-mixing effect for coherent sensing," Adv. in Optics and Photonics 7, 570 (2015).

[4] H. Matsumoto, "Alignment of length-measuring IR laser interferometer using laser feedback," Appl. Optics, 19, 1 (1980).

[5] A. Magnani, A. Pesatori, M. Norgia, "Self-Mixing Vibrometer with RealTime Digital Signal Elaboration", Appl. Optics 51, 5318 (2012).

[6] F. Gouaux, N. Sarvagent, and T. Bosch, "Absolute distance measurement with an optical feedback interferometer," Appl. Optics, 37, 6684 (1998).

[7] D. Melchionni, A. Magnani, A. Pesatori, M. Norgia, "Development of a design tool for closed-loop digital vibrometer", Appl. Optics 54, 9637 (2015).

[8] Y. Fan, Y. Yu, J. Xi, J. F. Chicharo, "Improving the measurement performance for a self-mixing interferometry-based displacement sensing system," Appl. Opt. 50, 5064 (2011).

[9] L.Scalise, Y.Yu, G. Giuliani, G. Plantier, and T. Bosch: "Self-Mixing Laser Diode Velocimetry: Application to Vibration and Velocity Measurement," IEEE Trans. on Instr. and Meas. 53, 223 (2004).
[10] U. Zabit, R. Atashkhooei, T. Bosch, S. Royo, F. Bony, and A. D. Rakic, "Adaptive self-mixing vibrometer based on a liquid lens," Opt. Lett. 35, $1278(2010)$.

[11] A. Magnani, D. Melchionni, A. Pesatori, M. Norgia, "Self-mixing digital closed-loop vibrometer for high accuracy vibration measurements", Optics Comm. 365, 133 (2016).

[12] G.Giuliani, M. Norgia, "Laser diode linewidth measurement by means of self-mixing interferometry," IEEE Phot. Techn. Lett., PTL-12,1028 (2000).

[13] Y. Yu, G. Giuliani, S. Donati, "Measurement of the linewidth enhancement factor of semiconductor lasers based on the optical feedback self-mixing effect," IEEE Photon. Tech. Lett. 16, 990, (2004).

[14] R. Atashkooei, J.C Urresty, S. Royo, J.R.Riba and L.Romeral, "Runout tracking in electric motors using self-mixing interferometry," IEEE Trans. Mechatronics, 19, 184 (2014).

[15] S. Donati, M. Norgia, and G. Giuliani, "Self-mixing differential vibrometer based on electronic channel subtraction," Appl. Opt. 45, 7264 (2006).

[16] R.Kliese, Y.L. Lim, T. Bosch, A. D. Rakić, "GaN laser self-mixing velocimeter for measuring slow flows," Optics Lett. 35, 814 (2010).

[17] A. G. Demir, P. Colombo, M. Norgia, B. Previtali, "Evaluation of selfmixing interferometry performance in the measurement of ablation depth," IEEE Trans. on Instr. and Meas. 65, 2621 (2016).

[18] S. Donati, M.Norgia, "Self-mixing Interferometry for Biomedical Signals Sensing," IEEE J. Select. Topics Quant. El. 20, DOI 10.1109 (2014).

[19] A.Arasanz, F.J.Azcona, S.Royo, A.Jha and J.Padellorens, "A new method for the acquisition of arterial pulse wave using self-mixing interferometry," Opt. and Laser Techn. 63, 98 (2014).

[20] H. Moench et al., "VCSEL based sensors for distance and velocity," Proc. SPIE 9766, Vertical-Cavity Surface-Emitting Lasers XX, 97660A (2016).

[21] M.B. Spencer, W.E. Lamb, "Laser with a Transmitting Window," Jr. Phys. Rev. A 5, 884 (1972).

[22] R. Lang and K. Kobayashi, "External Optical feedback Effects on Semiconductor Injection Laser Properties," IEEE J. Quantum Electron. 16, 347 (1980).

[23] E. M. Randone and S. Donati, "Self-mixing interferometer: analysis of the output signals," Opt. Express 14, 9788 (2006)

[24] K.Li, F.Cavedo, A.Pesatori, C. Zhao, M.Norgia, "Balanced detection for self-mixing interferometry," Opt. Lett. 42, 283 (2017).

[25] P.Dean, Y.L.Lim, A.Valavanis, R.Kleise, M.Nikolic, S.P.Khanna, M.Lachab, D.Indjin, Z.Ikonic, P.Harrison, A.D.Rakic, E.H.Lindfield, G.Davies, "Terahertz imaging through self-mixing in a quantum cascade laser," Opt. Lett. 36, 2587 (2011).

[26] S. Donati, "Responsivity and Noise of Self-Mixing Photodetection Schemes," IEEE J. of Quantum El., 47, 1428 (2011).

[27] V.Contreras, J.Lonnquist and J.Toivonen, "Edge filter enhanced selfmixing interferometry," Opt. Lett. 40, 2814 (2015).

[28] S.Donati: "Photodetectors", Prentice Hall, Upper Saddle River, NJ, USA (1999).

[29] G.Giuliani, S.Bozzi-Pietra, S. Donati, "Self-mixing laser diode vibrometer," Meas. Science and Techn. 14, 24 (2003).

[30] S.Donati, D.Rossi, M.Norgia: " Single Channel Self-Mixing Interferometer Measures Simultaneously Displacement and Tilt and Yaw Angles of a Reflective Target", IEEE Journal Quantum El., vol. QE-51, 2015, DOI 1400108.

[31] S.Donati: Electrooptical Instrumentation, Prentice Hall, Upper Saddle River, NJ, USA (2004). 